\title{
QUADRATIC EQUATIONS AND APPLICATIONS \\ TO CHANDRASEKHAR'S AND RELATED EQUATIONS
}

\author{
IOANNIS K. ARgYROS
}

\begin{abstract}
A new technique, using the contraction mapping theorem, for solving quadratic equations in Banach space is introduced. The results are then applied to solve Chandrasekhar's integral equation and related equations without the usual positivity assumptions.
\end{abstract}

\section{Introduction}

Consider the equation

$$
x=y+B(x, x)
$$

in a Banach space $X$ over the field $S$ of real numbers, where $B: X \times X+X$ is a bounded bilinear operator and $y \in X$ is fixed. We prove a consequence of the contraction mapping principle which can be used to prove existence and uniqueness of solutions of (1). For the special cases of Chandrasekhar's equation [5]:

$$
x(s)=1+\lambda x(s) \int_{0}^{1} \frac{s}{s+t} x(t) d t
$$

and the Anselone-Moore system [1]:

$x_{j}(s)=y_{j}(s)+\gamma \int_{0}^{1} L_{j 1}(s, t) x_{1}(t) x_{2}(t) d t+\gamma \int_{0}^{1} L_{j 2}(x, t) \frac{1}{2} x_{1}^{2}(t) d t, j=1,2$, (H)

Received 26 February 1985.

Copyright Clearance Centre, Inc. Serial-fee code: 0004-9727/85 $\$ A 2.00+0.00$. 
our theorem yields existence and uniqueness for larger values of the positive parameters $\lambda, \gamma$ than previously known in [1], [9], as well as providing more accurate information on the location of solutions.

More precisely we prove existence and uniqueness in a specific closed ball $\bar{U}(z, r)$ for a solution of (C) provided that

$$
\lambda<.424059
$$

and for a solution of (H) provided that

$$
\gamma<\left[4\|y\| \max _{j=1,2}\left(\left\|L_{j 1}\right\|+\frac{1}{2}\left\|L_{j 2}\right\|\right)\right]^{-1} .
$$

From [4], [5], [6], [7], [8], one can describe the existence theory of (C) completely for the case $\operatorname{Re}(\lambda)>0$, however, the techniques applied there are not as general as the one described here, because they make use among other assumptions (which hold only for the specific $B$ given in (C)) of the differentiability of the operator

$$
P(x)(s)=x-1-\lambda x(s) \int_{0}^{1} \frac{t}{s+t} x(t) d t
$$

and the invertibility of $P^{\prime}\left(x_{0}\right)$ for some specific $x_{0} \in C[0,1]$.

The principal new idea in our general theorem is the introduction of a second quadratic equation

$$
z=y+F(z, z)
$$

for comparison with (1). The estimates on $(C)$ and $(H)$ are then obtained under suitable choices of $F$.

Finally we show how we can use the solutions of finite rank equations to approximate solutions of (1) when $B$ is the uniform limit of finite rank operators.

Some of these results were announced in [2].

\section{Preliminaries}

DEFINITION 1. An operator $B: X \times Y \rightarrow Z$ sending $(x, y) \in X \times Y$ to $B(x, y) \in Z$ is called bilinear if it is linear in each variable separately 
and symmetric if $X=Y$ and $B(x, y)=B(y, x)$ for all $x \in X$.

DEFINITION 2. The mean $\bar{B}$ of $B$ on $X \times X$ is defined by

$$
\bar{B}(x, y)=\frac{1}{2}(B(x, y)+B(y, x)) \text { for all } x, y \in X .
$$

DEFINITION 3. An operator $Q: X \rightarrow Z$ sending $x \in X$ to $Q(x) \in Z$ is called quadratic if there exists a bilinear operator $B: X \times X \rightarrow Z$ such that

$$
Q(x)=B(x, x) \text { for all } x \in X
$$

The following proposition can now be easily proved.

PROPOSITION 1. An operator $Q: X \rightarrow Z$ is a quadratic operator if and only if there exists a symmetric bilinear operator $\bar{B}: X \times X \rightarrow Z$ satisfying

$$
Q\left(c_{1} x+c_{2} y\right)=c_{1}^{2} Q(x)+2 c_{1} \bar{B}(x, y)+c_{2}^{2} Q(y)
$$

for all $x, y \in X, c_{1}, c_{2} \in S$. Such a symmetric $\bar{B}$ is unique.

DEFINITION 4. A bilinear operator $B: X \times Y \rightarrow Z$ is said to be bounded if there exists $c>0$ such that

$$
\|B(x, y)\| \leq c\|x\|\|y\| \text { for al1 }(x, y) \in X \times Y \text {. }
$$

The quantity $\|B\|=\sup _{\|x\| \leq 1,\|y\| \leq 1}\|B(x, y)\|$ is called the norm of $B$.

DEFINITION 5. A quadratic operator $B: X \rightarrow Z$ is said to be bounded if there exists $c>0$ such that

$$
\|Q(x)\| \leq e\|x\|^{2} \text { for all } x \in X
$$

The quantity $|Q|=\sup _{|x| \leq 1}|Q(x)|$ is called the norm of $Q$.

From now on we assume that $X=Y=Z$ unless otherwise stated. 
3. A consequence of the contraction mapping principle, Rall's Theorem and equation (1)

We can assume without loss of generality that $B$ in (1) is symmetric since it agrees with $\bar{B}$ on the diagonal of $X \times X$.

We now prove a consequence of the contraction mapping principle for (1).

THEOREM 1. Let $B$ be a bounded bilinear operator on $X \times X$ and suppose $y$ and $z$ bezong to $X$. Define $T: X \rightarrow X$ by

$$
T(x)=y+B(x, x)
$$

set

$$
\begin{aligned}
& a=\frac{1}{2\|B\|}-\|z\|, \\
& b=a-\left(a^{2}-\frac{\|T(z)-z\|}{\|B\|}\right)^{1 / 2},
\end{aligned}
$$

and assume $b$ is nonnegative and $a \neq 0$. Then

(i) $T$ has a zonique fixed point in $U(z, a)=\{x \in X \mid\|x-z\|<\alpha\}$;

(ii) this fixed point actually lies in $\bar{U}(z, b)$.

Proof. The hypothesis, $b \geq 0$ and $a \neq 0$, implies that $a>0$ and

$$
a^{2}-\frac{\|T(z)-z\|}{\|B\|} \geq 0
$$

Fix $r$ such that $b \leq r<a$.

Claim 1. $T$ is a contraction operator on $\bar{U}(z, r)$. If $x_{1}, x_{2} \in \bar{U}(z, r)$, then it is routine to show

$$
\begin{aligned}
\left\|T\left(x_{1}\right)-T\left(x_{2}\right)\right\| & =\left\|B\left(x_{1}, x_{1}\right)-B\left(x_{2}, x_{2}\right)\right\| \\
& \leq 2(x+\|z\|)\|B\| x_{1}-x_{2} \| .
\end{aligned}
$$

set $q=2\left(r+\|\|^{\|}\right) B \|$. By hypothesis,

$$
r<\frac{1}{2\|B\|}-\|z\|
$$

so $0<q<1$ and the claim is proved. 
Claim 2. $T$ maps $\bar{U}(z, r)$ into $\bar{U}(z, r)$. We have

$$
\begin{aligned}
\|T(x)-z\| & =\|T(x)-T(z)+T(z)-z\| \\
& \leq\|B(x, x)-B(z, z)\|+\|T(z)-z\| \\
& \leq\|B\| r^{2}+2\|B\| \boldsymbol{z}\|r+\| T(z)-z \| .
\end{aligned}
$$

Define the real quadratic polyhomial $g(r)$ by

$$
g(r)=\|B\| r^{2}+(2\|B\|\|z\|-1) r+\|T(z)-z\| .
$$

To establish the claim we must show that $g(r) \leq 0$ for all $r, b \leq r<a$. Now the quadratic function $g(r)$ is convex, with smallest root at $b$ and minimum occurring at $a$. So for $b \leq r<a$,

$$
\|B\| r^{2}+2\|B\|\|z\| r+\|T(z)-z\| \leq r .
$$

The theorem now follows from the contraction mapping principle.

COROLLARY 1. If

$$
4\|B\|\|y\|<1
$$

then

(i) the equation

$$
x=y+B(x, x)
$$

has a unique solution $x$ in the open ball $U\left(0, x_{2}\right)$, where

$$
r_{2}=(2 \| B)^{-1}
$$

(ii) moreover $x \in \bar{U}\left(0, x_{1}\right)$, where

$$
r_{1}=\left[1-(1-4\|B\|\|y\|)^{1 / 2}\right](2\|B\|)^{-1} \text {. }
$$

Proof. Take $z=0$ in Theorem 1 .

We now state Rall's theorem for comparison. The proof can be found in [9].

THEOREM 2. If

$$
4 \mid B\|y\|<
$$

then 
(i) equation (1) has a solution $x \in X$ satisfying

$$
\|x\| \leq\left[1-(1-4\|B\|\|y\|)^{1 / 2}\right](2\|B\|)^{-1} ;
$$

(ii) moreover $x$ is unique in $U(x, R)$, where

$$
R=(1-4\|B\|\|y\|)^{1 / 2}(2\|B\|)^{-1} \text {. }
$$

Note that Theorem 2 and Corollary 1 provide the same estimate on $\|x\|$, but Theorem 2 guarantees uniqueness in $U(x, R)$ and not in $\bar{U}(0, r)$.

Corollary 1 is a crude application of Theorem 1 . Sometimes it is possible to introduce an auxiliary quadratic equation which is "close to" (I), but easier to handle. In the next section, we will use Theorem 1 in a more subtle way to exploit this idea. In particular, we will learn how to solve (1) in cases not covered by Rall's theorem.

\section{Auxiliary quadratic equations}

THEOREM 3. Consider the equation

$$
z=y+F(z, z)
$$

where $F: X \times X \rightarrow X$ is a bounded symetric bilinear operator and $y$ is fixed in $X$. Suppose that there exists a solution $z$ of (2) satisfying

$$
\|z\|<\left[2 \sqrt[\| B]{ } \|(\sqrt[\|B-F\|]{ }+\sqrt[\|B\|]{ }]^{-1} .\right.
$$

Then

(i) equation (1) has a unique solution $x \in U(z, a)$;

(ii) moreover, $x \in \bar{U}(z, b)$, where

$$
b=\left\{1-2\|B\|\|z\|-\left[(2\|B\|\|z\|-1)^{2}-4\|B-F\|\|B\|\|z\|^{2}\right]^{1 / 2}\right\}(2\|B\|)^{-1} .
$$

Proof. With $T$ as in Theorem 1, we have

$$
\begin{aligned}
\|T(z)-z\| & =\|(B-F)(z, z)+F(z, z)+y-z\| \\
& \leq\|(B-F)(z, z)\|+\|F(z, z)+y-z\| \\
& \leq\|B-F\|\left\|_{z}\right\|^{2} .
\end{aligned}
$$

So

$$
|T(z)-z| \leq|B-F| \|\left. z\right|^{2}
$$


Now (3) implies the hypothesis of Theorem 1 since

$$
\|z\|<(2\|B\|)^{-1} \Rightarrow a>0,
$$

while by (3) and (4) we have

$$
\frac{1}{2\|\bar{B}\|}-\|z\|>\sqrt{\frac{\mathbb{B}-F \|}{\|B\|}}\|z\|
$$

or

$$
a>\sqrt{\frac{\|T(z)-z\|}{\|B\|}} \Rightarrow b \geq 0
$$

The following generalization of Theorem 3 allows us to deal with the situation when $z$ is not an exact solution of (2).

THEOREM 4. Let $B$ and $F$ be bounded bilinear operators on $X \times X$ and suppose $y$ and $z$ belong to $X$. Define $T: X \rightarrow X$ by

$$
T(x)=y+B(x, x),
$$

and set

$$
\begin{aligned}
& a=\frac{1}{2\|B\|}-\|z\| \\
& \varepsilon=\|F(z, z)+y-z\|\|z\|^{-2} \\
& b=a-\left[a^{2}-\frac{\|B-F\|\|z\|^{2}+\varepsilon\left\|_{z}\right\|^{2}}{\|B\|}\right]^{1 / 2} .
\end{aligned}
$$

Assume that

$$
\|z\|<\left[2 \sqrt{\|B\|}(\sqrt{\|B\|}+\sqrt{B-F \|+\varepsilon)}]^{-1}\right.
$$

then

(i) $T$ has a unique fixed point in $U(z, a)$;

(ii) this fixed point actually lies in $\bar{U}(z, b)$.

Proof. Similar to Theorem 3.

We complete this section by recording certain facts concerning Theorem 3.

REMARK 1. The iteration 


$$
x_{n+1}=y+B\left(x_{n}, x_{n}\right), n=1,2, \ldots
$$

converges for any $x_{0} \in \bar{U}(z, b)$ to the solution $x$ of $(1)$ at the rate of a geometric progression with quotient

$$
q=1-\left[(2\|B\|\|z\|-1)^{2}-4\|B-F\|\|B\|\|z\|^{2}\right]^{1 / 2} .
$$

Proof. By Theorem 3 we have

$$
\begin{aligned}
q & =2(b+\|z\|)\|B\| \\
& =1-\left[(2\|B\|\|z\|-1)^{2}-4\|B\|\|B-F\|\|z\|^{2}\right]^{1 / 2} .
\end{aligned}
$$

PROPOSITION 2. Under the hypothesis of Theorem 3, the solution obtained in Theorem 3 satisfies

$$
\|x\|<(2\|B\|)^{-1} \text {. }
$$

Proof. By Theorem 3 we have

$$
\|x-z\|<a,
$$

so that

$$
\|x\| \leq\|z\|+a,
$$

that is, $\|x\|<(2\|B\|)^{-1}$, and the result follows.

COROLLARY 2. For any $y \in X$ such that $\|y\|<(4\|B\|)^{-1}$,

(i) equation (1) has a vonique solution $x \in U(y, a)$, where

$$
a=(1-2\|B\|\|y\|)(2\|B\|)^{-1} ;
$$

(ii) moreover $x \in \bar{U}(y, b)$, where

$$
b=\left[1-2\|B\| y \|-(1-4\|B\|\|y\|)^{1 / 2}\right](2\|B\|)^{-1} .
$$

Proof. Apply Theorem 3 with $F=0$ and $z=y$.

REMARK 2. Theorem 3 may be applicable even if the hypothesis in Corollary 1 or Theorem 2 is violated. Here is an example in $X=\mathbb{R}$.

EXAMPLE 1. Let

$$
\begin{array}{lll}
x=-.251+x^{2} & \text { for } & x=y+B(x, x) \quad \text { and } \\
z=-.251+.8 z^{2} & \text { for } & z=y+F(z, z) .
\end{array}
$$


PROPOSITION 3. Assume

(i) the hypotheses of Theorems 2, 3 and Corollary 1 are satisfied;

(ii) $(\|B\|-\|B-F\|)\|z\|^{2}-\|z\|+\|y\|>0$.

Then Theorem 3 provides a sharper estimate on $x$ than Theorem 2 or corollary 1.

Proof. By Theorem 3,

$$
\|x-z\| \leq b \text { so }\|x\| \leq b+\|z\| .
$$

By Theorem 2 and Corollary 1 ,

$$
\|x\| \leq\left[1-(1-4\|B\|\|y\|)^{1 / 2}\right](2\|B\|)^{-1}
$$

so it is enough to show

$$
\begin{aligned}
{\left[1-\left((2\|B\|\|z\|-1)^{2}-4\|B\|\|B-F\|\|z\|^{2}\right)^{1 / 2}\right](2\|B\|)^{-1} } \\
<\left[1-(1-4\|B\|\|y\|)^{1 / 2}\right](2\|B\|)^{-1}
\end{aligned}
$$

or

$$
(\|B\|-\|B-F\|) \quad\|z\|^{2}-\|z\|+\|y\|>0
$$

and the result now follows from (ii).

\section{Chandrasekhar's integral equation}

PROPOSITION 4. Take $X=C[0,1]$ with sup-norm and define the operator $K: X x X \rightarrow X$ by

$$
K(x, y)(s)=\frac{1}{2}\left[x(s) \int_{0}^{1} \frac{s}{s+t} y(t) d t+y(s) \int_{0}^{1} \frac{s}{s+t} x(t) d t\right]
$$

Then $\|K\|=1 n 2$.

Proof. The operator $Q: X \rightarrow X$ defined by

$$
Q(x)=x(s) \int_{0}^{1} \frac{s}{s+t} x(t) d t
$$

is a quadratic operator since

$$
K(x, x)=Q(x) \text { for all } x \in X .
$$

Now

$$
Q=\max _{s} \int_{0}^{1}\left|\frac{s}{s+t}\right| d t=\ln 2
$$


and since always

$$
\|Q\| \leq\|K\|
$$

we obtain

$$
\text { In } 2 \leq\|K\| .
$$

The proof will be completed if we prove that

$$
\|K\| \leq \ln 2 \text {. }
$$

But by the definition of $K$,

$$
\|K\| \leq \frac{1}{2} \max _{s}\left(2 \int_{0}^{1}\left|\frac{s}{s+t}\right| d t\right)=\ln 2
$$

and the result follows.

We now apply Theorem 2, Corollary 1 and 2 in (C) with $B=\lambda K$. According to Corollary 1 ,

(i) equation (1) has a unique solution $x \in U\left(z, r_{2}\right)$, where

$$
r_{2}=\left(\begin{array}{lll}
2 \lambda & \ln 2
\end{array}\right)^{-1}
$$

(ii) moreover $x \in \bar{U}\left(z, r_{1}\right)$, where

$$
r_{1}=\left[1-(1-4 \lambda \text { ln } 2)^{1 / 2}\right]\left(\begin{array}{lll}
2 \lambda & \text { ln } 2)^{-1},
\end{array}\right.
$$

provided that $4 \lambda$ in $2<1$, that is, $\lambda<.36067$.

Similarly according to Theorem 2,

(i) equation (1) has a unique solution in $U(x, R)$, where

$$
R=(1-4 \lambda \text { ln } 2)^{1 / 2}(2 \lambda \text { in } 2)^{-1}
$$

provided that $\lambda<.36067$.

Finally, according to Corollary 2,

(i) equation ( 1 ) has a unique solution $x \in U(1, a)$, where

$$
a=(1-2 \lambda \ln 2)(2 \lambda \text { In } 2)^{-1} \text {; }
$$

(ii) moreover $x \in \bar{U}(1, b)$, where

$$
b=\left[1-2 \lambda \text { In } 2-(1-4 \lambda \text { ln } 2)^{1 / 2}\right](2 \lambda \text { In } 2)^{-1} \text {. }
$$


Theorem 3 has the following corollary.

PROPOSITION 5. Consider the equation

$$
z=y+\lambda B(z, z) \text {. }
$$

Suppose

$$
2 \lambda\|B\| \rrbracket z \|<1
$$

where $z$ and $\lambda$ satisfy (5). Then for

$$
\lambda \leq \lambda_{1}<c_{1}
$$

with

$$
c_{1}=[4\|B\|\|z\|(1-\lambda\|B\|\|z\|)]^{-1},
$$

the conclusions of Theorem 3 for the equation

$$
x=y+\lambda_{1} B(x, x)
$$

hold.

Proof. To apply Theorem 3 we need to prove

$$
\left.\|z\|<\left[2\left(\lambda_{1}\left\|_{B}\right\|\right)^{1 / 2}\left(\left(\left|\lambda-\lambda_{1}\right|\|B\|\right)^{1 / 2}+\left(\lambda_{1}\left\|_{B}\right\|\right)^{1 / 2}\right)\right)\right]^{-1} .
$$

This is proved by using (6) and solving (7) for $\|z\|$.

In practice, an exact solution of the auxiliary equation (2) or (5) can seldom be obtained. The following proposition, whose proof is similar to that of Proposition 5, guarantees that the original equation (1) has a solution even when we can only find an approximate solution of (2) or (5).

PROPOSITION 6. Let $B$ be a bilinear operator on $X \times X$, suppose $y$ and $z$ belong to $X$ and $\lambda$ is a positive parameter. Set

$$
\varepsilon=\|\lambda B(z, z)+y-z\|\|B\|\|z\|^{-2}
$$

and

$$
c_{1}=\{4\|z\| B \|[1-(\lambda-\varepsilon)\|B\|\|z\|]\}^{-1}
$$

Then, for any $\lambda_{1}$ satisfying $\lambda \leq \lambda_{1}<c_{1}$, the equation

$$
x=y+\lambda_{1} B(x, x)
$$


has a unique solution $x$ in $U(z, a)$ and in fact this solution lies in $\bar{U}(z, b)$. Here

$$
\begin{aligned}
& a=\frac{1}{2 \lambda_{1}\left\|_{B}\right\|}-\left\|_{z}\right\| \\
& b=a-\left[a^{2}-\left(1-\frac{\lambda}{\lambda_{1}}\right)\left\|_{z}\right\|^{2}-\frac{\varepsilon}{\lambda_{1}}\left\|_{z}\right\|^{2}\right]^{1 / 2} .
\end{aligned}
$$

REMARK 3. According to Theorem 2 and Corollary 1 or 2 , Chandrasekhar's equation

$$
z(s)=1+\lambda K(z(s), z(s))=1+\lambda z(s) \int_{0}^{1} \frac{s}{s+t} z(t) d t
$$

has a solution for any $\lambda$ satisfying $\lambda<.36067$, but now using Proposition 6 we can extend the range of $\lambda$ to $\lambda<.424059$.

Here are some characteristic values for $\lambda$, the norm of the corresponding approximate solution $z_{n}$ and $c_{1}$.

\begin{tabular}{ccc}
\hline$\lambda$ & $\left\|z_{n}\right\|$ & $c_{1}$ \\
\hline .35 & 1.44474532 & .384363732 \\
$\vdots$ & $\vdots$ & $\vdots$ \\
.4 & 1.59821923 & .405244331 \\
$\vdots$ & $\vdots$ & $\vdots$ \\
.42 & 1.68363661 & .420163281 \\
$\vdots$ & $\vdots$ & $\vdots$ \\
.423 & 1.69644924 & .423011429 \\
$\vdots$ & $\vdots$ & $\vdots$ \\
.424 & 1.70085561 & .424070047 \\
$\vdots$ & $\vdots$ & $\vdots$ \\
.424059378 & 1.700973716 & .424059379 \\
.424059379 & 1.700973721 & .424059379 \\
\hline
\end{tabular}


The approximate solution $z_{n}$ was calculated using the iteration suggested in Remark 1 with $z_{0}(s)=1$ for $\lambda<.36067$. The convergence of the iteration is then guaranteed by corollary 2. When

$.36067 \leq \lambda_{0}<.424059$ then the initial approximation $z_{0}(s)$ for such a $\lambda_{1}$ was the approximate solution of (C) for a smaller $\lambda_{1}$, which was sufficient for the use of Proposition 6 . Simpson's rule was used for the numerical quadratures over $s$ in the range $0(0.05)(1.0)$. The results agree with those in [11] at least to six decimal places. Finally $c_{1}$ was calculated according to Proposition 6.

Numerical iteration [8] suggests that if $\lambda \geq .42406$ then $\|x\| \geq\left(2 \lambda\|K\|^{\|}\right)^{-1}$. This implies that if the estimate on $\|x\|$ given by Proposition 2 is the "best" that Theorem 4 can provide, then Proposition 6 provides the widest possible range for $\lambda$.

\section{Anselone-Moore's system}

A system of quadratic equations in $X$ of the form

$$
x_{i}=y_{i}+B_{i}(x, x), i=1,2, \ldots, n
$$

where $x_{i}, y_{i} \in X, x=\left(x_{1}, \ldots, x_{n}\right) \in X^{n}$ and $B_{i}: X x X+X, i=1,2, \ldots, n$ are bounded bilinear operators, can be viewed as a quadratic equation of the form

$$
x=y+B(x, x) \text { in } x^{n}
$$

where the norm of a vector in $x^{n}$ is defined by

$$
|x|=\max \left\{I_{x_{1}}\left|, \ldots, \mathbf{I}_{n}\right|\right\} ;
$$

and therefore

$$
|\tilde{B}|=\max \left\{\left|B_{1}\right|, \ldots,\left|B_{n}\right|\right\} .
$$

Let $X=C[0,1]$ and define the continuous linear operators $L_{j k}: X \rightarrow X$ by

$$
\left(L_{j k} f\right)(s)=\int_{0}^{1} N_{j k}(s, t) f(t) d t, 0 \leq s \leq 1,
$$


where the $N_{j k}, j, k=1,2$ are continuous functions of two variables. Define the quadratic operator $E: X \times X \rightarrow X \times X$ by

$$
E(x)=\left(\begin{array}{l}
L_{11} x_{1} x_{2}+\frac{1}{2} L_{12} x_{1}^{2} \\
L_{21} x_{1} x_{2}+\frac{1}{2} L_{22} x_{1}^{2}
\end{array}\right) \text { with } x=\left(x_{1}, x_{2}\right)
$$

Also define the operator $\tilde{B}: X \times X \times X \times X \rightarrow X \times X$ by

$$
\widetilde{B}(x, y)=\frac{1}{4}[E(x+y)-E(x-y)] .
$$

Note that $\tilde{B}$ is a bounded symmetric bilinear operator.

We now state for comparison Anselone-Moore's theorem concerning the solution of (H). The proof can be found in [1].

THEOREM 5. If

$$
4 \gamma\|y\|\|E\|<1
$$

and

$$
\|E\| \leq \max _{j=1,2}\left(\left\|L_{j 1}\right\|+\left\|L_{j 2}\right\|\right)
$$

then

(i) equation (H) has a unique solution $x \in U\left(0, r_{2}^{\prime}\right)$, where

$$
r_{2}^{\prime}=\left(2 \gamma \| E^{\|}\right)^{-1}
$$

(ii) moreover $x \in \bar{U}\left(0, r_{1}^{\prime}\right)$, where

$$
r_{1}^{\prime}=\left[1-(1-4 \gamma\|y\|\|E\|)^{1 / 2}\right](2 \gamma\|E\|)^{-1} \text {. }
$$

Equation (H) is of the form (9). Here, $X=C[0,1]$ and

$$
\|\tilde{B}\| \leq \max _{j=1,2}\left(\left\|L_{j 1}\right\|+\frac{1}{2}\left\|L_{j 2}\right\|\right)
$$

where

$$
\left\|L_{j k}\right\|=\sup _{s} \int_{0}^{1}\left|N_{j k}(s, t)\right| d t, j, k=1,2 .
$$

since

$$
\max _{j=1,2}\left(\left\|L_{j 1}\right\|+\frac{1}{2}\left\|L_{j 2}\right\|\right) \leq \max _{j=1,2}\left(\left\|L_{j 1}\right\|+\left\|L_{j 2}\right\|\right),
$$


Corollary 2 provides both a wider range for $\gamma$ and better information about where the solution lies than Theorem 5. Finally we can use Proposition 6 to extend the range of $\gamma$ even more, but we omit the details here.

\section{Quadratic finite rank operator equations}

DEFINITION 6. An operator $P: X \rightarrow Z$ sending $x \in X$ to $P(x) \in Z$ has finite ronk $n$ if the span of the range of $P$ has dimension $n$.

REMARK 4. The problem of solving the quadratic equation

$$
x=y+Q(x)
$$

when $Q$ is of rank $n$ can be translated to a finite dimensional one by making the substitution $z=x-y$ to obtain the equation

$$
z=Q(z+y)
$$

which shows that $z$ must lie in the range of $Q$. More precisely we state the following theorem. The proof can be found in [3].

THEOREM 6. The point $w \in X$ is a solution of (10) if and only if

$$
w=y+\sum_{i=1}^{n} \xi_{i} b_{i}
$$

where the vector $\left(\xi_{1}, \ldots, \xi_{n}\right) \in S^{n}$ is a solution of

$$
x_{i}=1_{i}+\sum_{i=1}^{n} a_{i} x_{i}+\sum_{i, j=1}^{n} b_{i j} x_{i} x_{j}, i=1,2, \ldots, n \text { in } s^{n}
$$

where $1_{i}, a_{i}, b_{i, j}, i, j=1,2, \ldots, n$ are specific numbers depending on $Q$ and $y$.

REMARK 5. The linear part in equation (11) can sometimes be eliminated and therefore (11) can be written as

$$
x_{i}=1 !+\sum_{i, j=1}^{n} b_{i j}^{\prime} x_{i} x_{j}, i=1,2, \ldots, n \text { in } s^{n} .
$$

THEOREM 7. Consider the quadratic equations

$$
z=y+F_{n}(z, z)
$$


where $F_{n}: X x X+X, n=1,2, \ldots$ are bounded symmetric bilinear operators. If

(i) the sequence $\left\{F_{n}\right\}$ converges to $B$ uniformly as $n \rightarrow \infty$,

(ii) for each $n$ there exists $z_{n}$, satisfying (12) and $\sup _{z} \|<\left(2\|\|^{\|}\right)^{-1}$,

then the sequence $\left\{z_{n}\right\}$ converges to a solution $z$ of (1).

(Note that $B$ and the $F_{n}$ 's are not necessarily of finite rank here.)

Proof. We have

$$
z_{m}-z_{n}=F_{m}\left(z_{m}, z_{m}\right)-F_{n}\left(z_{n^{\prime}} z_{n}\right)
$$

by adding and subtracting $F_{n}\left(z_{m}, z_{m}\right), F_{m}\left(z_{n}, z_{n}\right), F_{n}\left(z_{m}, z_{n}\right)$ and $F_{m}\left(z_{m}, z_{n}\right)$ and rearranging we obtain

$$
\begin{aligned}
z_{m}-z_{n}=\left(F_{m}-E_{n}\right)\left(z_{m^{\prime}} z_{m}\right) & +\left(F_{m}-F_{n}\right)\left(z_{n}, z_{n}\right)+F_{n}\left(z_{m}, z_{m}-z_{n}\right) \\
& +F_{m}\left(z_{m}-z_{n}, z_{n}\right)+\left(F_{n}-F_{m}\right)\left(z_{m^{\prime}} z_{n}\right)
\end{aligned}
$$

Now, since $\sup _{n} z_{n} \|<\left(2\left\|_{B}\right\|\right)^{-1}$ there exists $c>0$ such that

$$
\left\|_{n}\right\| \leq c<\left(2^{\|} \|\right)^{-1}, n=1,2, \ldots,
$$

Moreover using (14) and the triangle inequality in (13) and setting $p=\lim ^{\|} z_{n}-z_{m} \|$ as $n, m \rightarrow \infty$ we obtain

$$
p \leq 2^{\|} B \| c p
$$

so

$$
p\left(2^{\|} \|_{C-1}\right) \geq 0,
$$

by (14) and (15), which implies $p=0$, that is,

$$
\lim ^{l l} z_{n}-z_{m} \|=0 \text { as } n \rightarrow \infty \text {. }
$$

Therefore the sequence $\left\{z_{n}\right\}, n=1,2, \ldots$ is a Cauchy sequence in a Banach space and as such it converges to some $z \in X$. The element $z \in X$ is a solution of (1) since

$$
z=\lim _{n \rightarrow \infty} z_{n}=\lim _{n \rightarrow \infty}\left(y+F_{n}\left(z_{n}, z_{n}\right)\right)
$$




$$
=y+\lim _{n \rightarrow \infty} F_{n}\left(z_{n}, z_{n}\right)=y+B(z, z) .
$$

Here we provide an example for Theorem 7 in $X=\mathbb{R}$ :

EXAMPLE 2. Let

$$
x=0+x^{2} \quad \text { for } \quad x=y+B(x, x)
$$

and

$$
z=0+\frac{n-1}{n} 2 \text { for } z=y+F_{n}(z, z)
$$

Then since

$$
\frac{n-1}{n} \rightarrow 1 \text { as } n \rightarrow \infty
$$

and

$$
z_{n}=0 \text { is a solution of (17) for each } n, n=1,2, \ldots
$$

the conditions of Theorem 7 are satisfied. Therefore since

$$
z_{n} \rightarrow 0 \text { as } n \rightarrow \infty, 0 \text { is a solution of (16). }
$$

The results presented here have been extended (3) to the more general equation

$$
x=y+L(x)+B(x, x)
$$

where $L: X \rightarrow X$ is a bounded linear operator.

\section{References}

[1] P.M. Anselone and R.H. Moore, "An extension of the NewtonKantorovich method for solving nonlinear equations", Tech. Rep. No. 520, U.S. Army Mathematics Research Center, University of Wisconsin, Madison (1965).

[2] I.K. Argyros, "On a contraction theorem and applications (to appear in Proceedings of Symposia in Pure Mathematics of the American Mathematical Societyl. 
[3] I.K. Argyros, "Quadratic equations in Banach space, perturbation techniques and applications to Chandrasekhar's and related equations. Ph.D. dissertation, University of Georgia, Athens (1984).

[4] R.I. Bowden and P.F. Zweifel, Astrophysics J. (1976), 219-232.

[5] S. Chandrasekhar, Radiative Transfer, Dover Pub1., New York, 1960.

[6] C.T. Kelley, "Solution of the Chandrasekhar H equation by Newton's method, J. Math. Phys. 21 (1980), 1625-1628.

[7] C.T. Kelley, "Approximation of some quadratic integral equations in transport theory", J. Integral Equations 4 (1982), 221-237.

[8] T.W. Mullikin, "Some probability distributions for neutron transport in a half-space", J. Applied Probability 5 (1968), $357-374$.

[9] L.B. Rall, "Quadratic equations in Banach space", Rend. Cire. Math. PaZermo 10 (1961), 314-332.

[10] L.B. Rall, Computational Solution of Nonlinear Operator Equations, John Wiley Publ., New York, 1968.

[11] D.W.N. Stibbs and R.E. Weir, "On the H-function for isotopic scattering, Monthly Not. Roy. Astron. Soc. 119 (1959), 515-525.

Department of Mathematics,

University of Iowa,

IOWA CITY, IA 52242 .

U.S.A. 